\title{
xCT, not just an amino-acid transporter: a multi-functional regulator of microbial infection and associated diseases
}

\author{
Lu Dai ${ }^{1,2}$, Mairi C. Noverr ${ }^{3}$, Chris Parsons ${ }^{2}$, Johnan A. R. Kaleeba ${ }^{4}$ and Zhiqiang Qin ${ }^{1,5 *}$ \\ Research Center for Translational Medicine and Key Laboratory of Arrhythmias of the Ministry of Education of China, East Hospital, Tongji University School of \\ Medicine, Shanghai, China \\ ${ }^{2}$ Department of Medicine, Louisiana State University Health Sciences Center, Louisiana Cancer Research Center, New Orleans, LA, USA \\ ${ }^{3}$ Department of Oral and Craniofacial Biology, Louisiana State University Health Sciences Center, Louisiana Cancer Research Center, New Orleans, LA, USA \\ ${ }^{4}$ Department of Microbiology and Immunology, Uniformed Services University of the Health Sciences, Bethesda, MD, USA \\ ${ }^{5}$ Department of Microbiology, Immunology, and Parasitology, Louisiana State University Health Sciences Center, Louisiana Cancer Research Center, New Orleans, \\ LA, USA
}

\section{Edited by:}

Evangelos Giamarellos-Bourboulis,

University of Athens, Greece

\section{Reviewed by:}

Paras Jain, Albert Einstein College of Medicine, USA

Aikaterini Pistiki, University General Hospital ATTIKO, Greece

*Correspondence:

Johnan A. R. Kaleeba, Department of Microbiology and Immunology, Uniformed Services University of the Health Sciences, 4301 Jones Bridge Road, Bethesda, MD 20814, USA

e-mail:kaleebajar@gmail.com:

Zhiqiang Qin, Department of Microbiology, Immunology, and

Parasitology, Louisiana State University Health Sciences Center, Louisiana Cancer Research Center, Room 743, 1700 Tulane Avenue,

New Orleans, LA 70112, USA

e-mail: zqin@Isuhsc.edu
Expression of $\mathrm{xCT}$, a component of the $\mathrm{x}_{\mathrm{c}}{ }^{-}$amino-acid transporter, is essential for the uptake of cystine required for intracellular glutathione (GSH) synthesis and maintenance of the intracellular redox balance. Therefore, $\mathrm{xCT}$ plays an important role not only in the survival of somatic and immune cells, but also in other aspects of tumorigenesis, including the growth and malignant progression of cancer cells, resistance to anticancer drugs, and protection of normal cells against oxidative damage induced by carcinogens. $\mathrm{XCT}$ also functions as a factor required for infection by Kaposi's sarcoma-associated herpesvirus (KSHV), the causative agent of Kaposi's sarcoma (KS) and other lymphoproliferative diseases associated with HIV/AIDS. In spite of these advances, our understanding of the role of $\mathrm{XCT}$ in the pathogenesis of infectious diseases is still limited. Therefore, this review will summarize recent findings about the functions of XCT in diseases associated with microbial (bacterial or viral) infections, in particular KSHV-associated malignancies. We will also discuss the remaining questions, future directions, as well as evidence that supports the potential benefits of exploring system $\mathrm{x}_{\mathrm{c}}{ }^{-}$as a target for prevention and clinical management of microbial diseases and cancer.

Keywords: KSHV, virus, bacteria, xCT, cysteine, glutamate

\section{BACKGROUND}

The $\mathrm{x}_{\mathrm{c}}{ }^{-}$amino-acid transporter, consisting of $\mathrm{xCT}$ (also named as SLC7A11) and its chaperone CD98, functions as a $\mathrm{Na}^{+}$-independent electroneutral exchange system for cystine/glutamate (Bannai and Kitamura, 1980). Expression of xCT on the cell membrane is essential for the uptake of cystine required for synthesis of intracellular glutathione (GSH), an anti-oxidant that plays an important role in maintaining the intracellular redox balance (Bannai, 1986; Patel et al., 2004). For most cancer cells, the uptake of cystine/cysteine from the microenvironment is crucial for growth and viability (Lo et al., 2008). Therefore, $\mathrm{xCT}$ is highly expressed by a variety of malignant tumors such as lymphoma, glioma, breast carcinoma, and prostate cancer (Gout et al., 2001; Narang et al., 2003; Chung et al., 2005; Doxsee et al., 2007). xCT is also involved in other important cellular functions within cancer cells, such as chemoresistance (Okuno et al., 2003; Huang et al., 2005) and autophagy (Guo et al., 2011). Interestingly, xCT-deficient mice display impaired survival of activated macrophages at the inflammatory site, which subsequently enhances chemically induced tumorigenesis (Nabeyama et al.,
2010). In addition, $\mathrm{xCT}$ has been associated with some central nervous system (CNS) and eye diseases, such as cerebral ischemia (stroke), trauma, Alzheimer's disease and retinopathy (Choi, 1987; Bridges et al., 2004; Nagasawa et al., 2005; Qin et al., 2006). Interestingly, we and others recently reported the functional contributions of $\mathrm{xCT}$ to the pathogenesis of Kaposi's sarcomaassociated herpesvirus (KSHV; Kaleeba and Berger, 2006a,b; Qin et al., 2010b, 2013; Dai et al., 2014a), a principal causative agent of several cancers arising in patients with compromised immune systems (Chang et al., 1994). In light of the emerging pleiotropic functions of $\mathrm{xCT}$, the goal of this review is to summarize recent findings about the role of $\mathrm{xCT}$ in microbial infection and associated diseases (particularly virus-associated malignancies), and to discuss potential future directions for research in this particular field.

\section{XCT AND KSHV PATHOGENESIS XCT AS A CELLULAR RECEPTOR FOR KSHV ENTRY}

Like other well-studied human herpesviruses, KSHV is presumed to enter cells by direct fusion with the target cell membrane as 
the predominant mode of infection (Dezube et al., 2002; Pertel, 2002; Kaleeba and Berger, 2006a; Longnecker and Neipel, 2007), although endocytosis and other receptor-independent mechanisms of entry have also been described (Akula et al., 2003; Rappocciolo et al., 2008; Greene and Gao, 2009; Garrigues et al., 2014). Using a functional complementary DNA selection assay, we recently identified $\mathrm{xCT}$ as an important component of a multimolecular complex that mediates KSHV entry by direct fusion with the target cell membrane (Kaleeba and Berger, 2006b). The KSHV receptor complex consists of xCT, CD98, and other "outside-in" signaling molecules such as integrins, suggesting that the virus also exploits pre-existing signaling pathways to initiate events that promote virion entry into a variety of cell types. Depending on the cell type, the initial stages of KSHV infection involve target cell recognition through temporal interactions with attachment factors (heparan sulfate and DC-SIGN) or signaling molecules such as integrins $(\alpha v \beta 3, \alpha 3 \beta 1$, and $\alpha v \beta 5)$; in some cases, these initial steps may be sufficient to mediate virion uptake, or they may be followed by recruitment of the CD98/xCT complex that then mediates viral glycoprotein-mediated fusion of the viral and target cell membranes (Veettil et al., 2008, 2014; Hahn et al., 2009). While these discoveries have advanced our understanding of the early events in the infectious process of $\mathrm{KSHV}$, several questions remain. For example, although recent studies have demonstrated that KSHV can infect some B cell subsets or cell-lines (Rappocciolo et al., 2008; Hassman et al., 2011; Myoung and Ganem, 2011; Dollery et al., 2014), we found that most monocytic and other immortalized lymphoblastoid cell lines did not express detectable levels of xCT mRNA and are generally refractory to $\mathrm{KSHV}$ infection. We also failed to detect $\mathrm{xCT}$ mRNA in human $\mathrm{CD}_{1}{ }^{+}$primary B cells isolated from fresh peripheral blood mononuclear cells (PBMCs), which is paradoxical given that the virus is etiologically linked to two B cell lymphoproliferative disorders, namely multicentric Castleman's disease (MCD) and primary effusion lymphoma (PEL; Cesarman et al., 1995; Soulier et al., 1995). Remarkably, ectopic expression of recombinant $\mathrm{xCT}$ does not cure the poor target susceptibility of B cell lines to KSHV, whereas over-expression of $\mathrm{xCT}$ in $\mathrm{K} 562$ cells and other cells that express $\alpha 3, \beta 1, \alpha \mathrm{v}$, or $\beta 3$ integrin subunits can render these cells more susceptible to KSHV, suggesting that the receptor function of $\mathrm{xCT}$ is more demonstrably robust in adherent and other cells that also express the relevant integrin co-receptor complexes that provide the requisite supporting role for efficient virion adsorption and signaling prior to virion entry. Interestingly, a recent study found that activated blood and tonsillar B cells can be productively infected with KSHV, which can be blocked by the pretreatment of the cells with antibody specific for DC-SIGN or with the selective DC-SIGN inhibitor, mannan, but not antibody specific for xCT (Rappocciolo et al., 2008). We also recently demonstrated that heparan sulfate (HS), not xCT, may be required for KSHV infection of human primary oral fibroblasts (Dai et al., 2014b). Together, these findings suggest that the receptor function of xCT may be cell-context dependent and is presumably determined by physiologic triggers of receptor expression within the target site, as well as the relative surface expression of various cell-type specific cofactors and other accessory molecules that must be recruited to the virion attachment site.

\section{xCT AND SURVIVAL OF KSHV-INFECTED CELLS}

The biological relevance of $\mathrm{xCT}$ in $\mathrm{KSHV}$ pathogenesis goes beyond its function as a virus receptor. As mentioned above, $\mathrm{xCT}$ naturally functions as a transporter for extracellular cystine that is then used in the synthesis of GSH, an anti-oxidant that restores the intracellular redox balance and protects cells from death induced by reactive oxygen species (ROS) or reactive nitrogen species (RNS; Bannai, 1986). As an adaptive response, $\mathrm{xCT}$ is upregulated when intracellular levels of GSH are low, such as in settings of oxidative stress among late stage HIV-AIDS patients who are also likely to experience more aggressive KS (Mallery et al., 2004; Ma et al., 2009, 2013). Accordingly, we found that $\mathrm{xCT}$ is upregulated within more advanced KS lesions that also express high levels of the KSHV latency-associated nuclear antigen (LANA) compared to early-stage KS lesions (Qin et al., 2010b). In the same study, we found that KSHV encodes multiple microRNAs that directly target $\mathrm{BACH}-1$, a negative transcriptional regulator of $\mathrm{xCT}$ that binds to the cis-acting "Antioxidant Response Element" (ARE) within the $x C T$ promoter region, thereby upregulating the receptor, which in turn facilitates viral dissemination and enhanced survival of virus-infected cells in the host microenvironment (Qin et al., 2010b). Our findings of a role for $\mathrm{KSHV}$ in regulation of its own receptor were the first to demonstrate a direct link between $\mathrm{xCT}$ receptor usage and $\mathrm{KSHV}$ pathogenesis, and have inspired ongoing work aimed at defining post-entry interactions of KSHV with the human host that can be explored as viable therapeutic targets for clinical management of $\mathrm{KS}$ and other virus-associated cancers.

By virtue of its role in maintaining the intracellular redox balance, $\mathrm{xCT}$ also protects KSHV-infected cells from death induced by RNS and other insults. Indeed, RNAi silencing of xCT expression impairs the resistance of KSHV-infected mouse macrophage RAW cells to death induced by the nitric oxide (NO) donor, Snitroso-N-acetylpenicillamine (SNAP; Qin et al., 2010b). We and others also recently reported that overexpression of $\mathrm{xCT}$ induces upregulation of 14-3-3 $\beta$ (a downstream regulatory protein from KSHV-infected cells and KS lesions), resulting in intracellular signal transduction via MAPK and increased cytokine release, cell growth, and invasiveness (Zeng et al., 2010; Qin et al., 2013). By enhancing biosynthesis of intracellular GSH, xCT also protects cancer cells from drug-induced oxidative stress by mediating detoxification and extrusion of chemotherapeutic drugs via its biophysical interactions with multidrug resistance proteins (Haimeur et al., 2002; Okuno et al., 2003; Filipits et al., 2005; Yang et al., 2006). For example, GSH induces a conformational change in the multidrug resistance-associated protein-1 (MRP1), which impairs its interaction with one of the most commonly used chemotherapeutic drugs, doxorubicin, and consequently reduces its drug-efflux function (Manciu et al., 2003).

Interestingly, the xCT/CD98 cystine transporter tightly associates with a multi-molecular "supercomplex" on the cell-surface that also includes Emmprin (CD147), LYVE-1 (a hyaluronan receptor), and BCRP (a drug-efflux pump protein responsible for multidrug resistance of KSHV-infected PEL cells; Qin et al., 2011). Within this complex, Emmprin has been reported to confer resistance to some chemotherapeutic drugs (Okuno et al., 2003; Yang et al., 2007; Zou et al., 2007). Indeed, expression of xCT 
in a panel of cancer cell lines has been associated with potency of 1,400 candidate anticancer drugs, including cisplatin (Huang et al., 2005). Since KSHV induces expression of both xCT and Emmprin in a variety of infected cell lines including PEL cells (Qin et al., 2010a,b; Dai et al., 2012, 2014a), it is probable that stabilization of the $\mathrm{xCT/CD98/CD147} \mathrm{supercomplex} \mathrm{plays}$ a critical role not only in intracellular energy metabolism (Xu and Hemler, 2005) but also in potentiating the efflux functions of multidrug transporters, which could enhance survival of KSHVassociated cancers including PEL.

\section{xCT AS A THERAPEUTIC TARGET FOR KSHV-ASSOCIATED LYMPHOMA}

As mentioned above, KSHV is a principal causative agent of PEL, which comprises transformed B cells harboring viral episomes. PEL is a rapidly progressing malignancy that arises preferentially within the pleural or peritoneal cavities of patients infected with HIV (Cesarman et al., 1995), with a median survival time of approximately 6 months even under conventional chemotherapy (Chen et al., 2007). xCT is highly expressed in a variety of KSHV-infected PEL cell-lines, and targeting xCT by either RNAi or selective inhibitors induces significant cell apoptosis potentially through regulation of host and viral factors including: (i) reducing intracellular GSH, (ii) increasing ROS, (iii) repressing cell-proliferation-related signaling, and (iv) inducing viral lytic gene expression (Dai et al., 2014a). We also demonstrated that an xCT selective inhibitor, Sulfasalazine (SASP), which has been approved by FDA for treatment of some inflammatory diseases (Gout et al., 2001), can effectively prevent PEL tumor progression in an immune-deficient xenograft mouse model (Dai et al., $2014 \mathrm{a}, \mathrm{c}$ ), supporting the potential benefit of targeting $\mathrm{xCT}$ as a strategy for attenuating progression to overt lymphomagenesis. With this as an important goal, additional studies are warranted in order to identify other cell-proliferation/growth-related factors that could be impacted by selective targeting of $\mathrm{xCT}$ (including those involved in cell-cycle and autophagy) not only within PEL cells but also in Burkitt's lymphoma (BL)-derived cell lines where xCT is also highly expressed (Dai et al., 2014a).

\section{XCT AND HIV PATHOGENESIS}

It has been reported that the HIV-encoded transactivator protein Tat can upregulate $\mathrm{xCT}$ expression within human primary microglia, retinal pigment epithelium and in retina from Tattransgenic mice (Bridges et al., 2004; Gupta et al., 2010; Pang et al., 2013), although the underlying mechanisms remain largely unknown. Increased expression of xCT within ocular tissue results in increased release of excitotoxic glutamate in exchange for extracellular cystine, which contributes to the excitotoxicity associated with non-infectious AIDS retinopathy (Bridges et al., 2004; Gupta et al., 2010). In this respect, the direct link between the amino acid transport activity of xCT and retinopathy among $\mathrm{HIV}$-infected individuals suggests that targeting xCT may represent a promising new strategy for ameliorating this disease in high-risk groups (Bridges et al., 2004; Butler and Thorne, 2012).

\section{xCT AND BACTERIAL INFECTION}

The $\mathrm{xCT}$-anchored $\mathrm{x}_{\mathrm{C}}{ }^{-}$cystine/glutamate transporter plays an important role not only in viral pathogenesis but also during bacterial infection. Thus, $\mathrm{xCT}$ mRNA and the cystine/glutamate transporter activity are dramatically induced in mouse peritoneal macrophages in vitro by bacterial lipopolysaccharide (LPS) even at very low concentrations, similar to that observed in the plasma of patients with sepsis (Sato et al., 1995). The same group further confirmed that ambient oxygen tension and/or oxidative stress are required for LPS-induced xCT expression and cystine/glutamate transport in mouse peritoneal macrophages in vitro (Sato et al., 2001). In an experimental endotoxemia mouse model, $\mathrm{xCT}$ mRNA was constitutively expressed in the brain, thymus, and spleen, and xCT mRNA was strongly upregulated in thymus and spleen following administration of a sublethal dose of LPS, indicating that $\mathrm{xCT}$ plays an important role during the host inflammatory response to bacterial infection in vivo (Taguchi et al., 2007). Interestingly, LPS induction of xCT expression is not mediated by the ARE in the promoter region as described previously (Sasaki et al., 2002); as such, the underlying mechanism for LPS-induced $\mathrm{xCT}$ expression requires further investigation.

\section{CONCLUDING REMARKS}

In contrast to extensive studies of the role of $\mathrm{xCT}$ as an amino-acid transporter involved in the survival of normal and cancer cells, there are very limited data describing its role in microbial infection and/or the pathogenesis of associated diseases. According to current data, at least $16.1 \%$ of cancers worldwide are linked to infectious diseases, and in Sub-Saharan Africa, this rate is almost 32.7\% (de Martel et al., 2012). Nonetheless, we note that although the preponderance of infectious cancer is associated with viruses, Helicobacter pylori, Mycobacterium, and some parasites have also been associated with a direct or potentiating role in development of some cancers as well (Fried et al., 2011; Pallis and Syrigos, 2013; Mesri et al., 2014; Park et al., 2014). In many cases, studies of the molecular mechanisms by which these infections contribute to the defining markers of cancer initiation and progression have yielded important insights into strategies for control and prevention of infectious disease. More specifically, recent findings from our group and others about the role of $\mathrm{xCT}$ in the pathogenesis of KSHV (a model oncogenic virus) have revealed the multi-functional role of $\mathrm{xCT}$ not only during primary infection but also in post-entry events that promote infected cell survival and tumorigenesis. Moreover, additional data demonstrating the biologic function of $\mathrm{xCT}$ in HIV and bacterial infection further illustrate the broad regulatory role of this protein in various infectious diseases. Therefore, to better understand the breadth of the xCT functional "network", it will be essential to elucidate: (i) the factors that regulate its expression in various cell types, (ii) the cognate proteins that interact with $\mathrm{xCT}$ on cell membrane in response to engagement by exogenous pathogens, (iii) the downstream genes that are controlled by signals initiated by these interactions at the cell surface, and (iv) whether xCT expression in tumors correlates with disease progression and resistance to chemotherapy in cancer-bearing individuals. New information derived from these studies will guide approaches for clinical management of xCT-associated abnormalities, including the development of more specific pharmacological methods for inhibiting or inducing system $\mathrm{x}_{\mathrm{c}}{ }^{-}$activity as a means for modulating progression of associated disease. With respect to PEL, cell-culture 
systems such as Nb2-SFJCD1 lymphoma cells (Gout et al., 2001) could be used as platforms for screening small molecule leadcompound libraries for novel xCT inhibitors. Along these lines, it is worth noting that some $\mathrm{xCT}$ inhibitors such as SASP have already been approved by FDA for treatment of certain infectious diseases, and its anti-cancer effects are being evaluated in clinical trials (Gout et al., 2001; Robe et al., 2006; Sontheimer and Bridges, 2012).

\section{ACKNOWLEDGMENTS}

This work was supported by grants from a Center for Biomedical Research Excellence P20-GM103501 subaward (RR021970), the Ladies Leukemia League Grant (2014-2015), and the National Natural Science Foundation (NNSF) of China (81101791, 81272191, 81472547, and 81400164).

\section{REFERENCES}

Akula, S. M., Naranatt, P. P., Walia, N. S., Wang, F. Z., Fegley, B., and Chandran, B. (2003). Kaposi's sarcoma-associated herpesvirus (human herpesvirus 8) infection of human fibroblast cells occurs through endocytosis. J. Virol. 77, 7978-7990. doi: 10.1128/JVI.77.14.7978-7990.2003

Bannai, S. (1986). Exchange of cystine and glutamate across plasma membrane of human fibroblasts. J. Biol. Chem. 261, 2256-2263.

Bannai, S., and Kitamura, E. (1980). Transport interaction of L-cystine and Lglutamate in human diploid fibroblasts in culture. J. Biol. Chem. 255, 23722376.

Bridges, C. C., Hu, H., Miyauchi, S., Siddaramappa, U. N., Ganapathy, M. E., Ignatowicz, L., et al. (2004). Induction of cystine-glutamate transporter $\mathrm{x}_{\mathrm{C}}$ - by human immunodeficiency virus type 1 transactivator protein tat in retinal pigment epithelium. Invest. Ophthalmol. Vis. Sci. 45, 2906-2914. doi: 10.1167/iovs.03-1355

Butler, N. J., and Thorne, J. E. (2012). Current status of HIV infection and ocular disease. Curr. Opin. Ophthalmol. 23, 517-522. doi: 10.1097/ICU.0b013e328358ba85

Cesarman, E., Chang, Y., Moore, P. S., Said, J. W., and Knowles, D. M. (1995). Kaposi's sarcoma-associated herpesvirus-like DNA sequences in AIDSrelated body-cavity-based lymphomas. N. Engl. J. Med. 332, 1186-1191. doi: 10.1056/NEJM199505043321802

Chang, Y., Cesarman, E., Pessin, M. S., Lee, F., Culpepper, J., Knowles, D. M., et al. (1994). Identification of herpesvirus-like DNA sequences in AIDS-associated Kaposi's sarcoma. Science 266, 1865-1869. doi: 10.1126/science.7997879

Chen, Y. B., Rahemtullah, A., and Hochberg, E. (2007). Primary effusion lymphoma. Oncologist 12, 569-576. doi: 10.1634/theoncologist.12-5-569

Choi, D. W. (1987). Ionic dependence of glutamate neurotoxicity. J. Neurosci. 7, 369-379.

Chung, W. J., Lyons, S. A., Nelson, G. M., Hamza, H., Gladson, C. L., Gillespie, G. Y., et al. (2005). Inhibition of cystine uptake disrupts the growth of primary brain tumors. J. Neurosci. 25, 7101-7110. doi: 10.1523/JNEUROSCI.5258-04.2005

Dai, L., Cao, Y., Chen, Y., Parsons, C., and Qin, Z. (2014a). Targeting xCT, a cystineglutamate transporter induces apoptosis and tumor regression for KSHV/HIVassociated lymphoma. J. Hematol. Oncol. 7, 30. doi: 10.1186/1756-8722-7-30

Dai, L., Defee, M. R., Cao, Y., Wen, J., Wen, X., Noverr, M. C., et al. (2014b). Lipoteichoic acid (LTA) and lipopolysaccharides (LPS) from periodontal pathogenic bacteria facilitate oncogenic herpesvirus infection within primary oral cells. PLoS ONE 9:e101326. doi: 10.1371/journal.pone.0101326

Dai, L., Trillo-Tinoco, J., Bai, L., Kang, B., Xu, Z., Wen, X., et al. (2014c). Systematic analysis of a xenograft mice model for $\mathrm{KSHV}^{+}$primary effusion lymphoma (PEL). PLoS ONE 9:e90349. doi: 10.1371/journal.pone.0090349

Dai, L., Qin, Z., Defee, M., Toole, B. P., Kirkwood, K. L., and Parsons, C. (2012). Kaposi sarcoma-associated herpesvirus (KSHV) induces a functional tumor-associated phenotype for oral fibroblasts. Cancer Lett. 318, 214-220. doi: 10.1016/j.canlet.2011.12.019

de Martel, C., Ferlay, J., Franceschi, S., Vignat, J., Bray, F., Forman, D., et al. (2012). Global burden of cancers attributable to infections in 2008: a review and synthetic analysis. Lancet Oncol. 13, 607-615. doi: 10.1016/S1470-2045(12)70137-7
Dezube, B. J., Zambela, M., Sage, D. R., Wang, J. F., and Fingeroth, J. D. (2002). Characterization of Kaposi sarcoma-associated herpesvirus/human herpesvirus- 8 infection of human vascular endothelial cells: early events. Blood 100, 888-896. doi: 10.1182/blood.V100.3.888

Dollery, S. J., Santiago-Crespo, R. J., Kardava, L., Moir, S., and Berger, E. A. (2014). Efficient infection of a human B cell line with cell-free Kaposi's sarcomaassociated herpesvirus. J. Virol. 88, 1748-1757. doi: 10.1128/JVI.03063-13

Doxsee, D. W., Gout, P. W., Kurita, T., Lo, M., Buckley, A. R., Wang, Y., et al. (2007). Sulfasalazine-induced cystine starvation: potential use for prostate cancer therapy. Prostate 67, 162-171. doi: 10.1002/pros.20508

Filipits, M., Pohl, G., Rudas, M., Dietze, O., Lax, S., Grill, R., et al. (2005). Clinical role of multidrug resistance protein 1 expression in chemotherapy resistance in early-stage breast cancer: the Austrian Breast and Colorectal Cancer Study Group. J. Clin. Oncol. 23, 1161-1168. doi: 10.1200/JCO.2005. 03.033

Fried, B., Reddy, A., and Mayer, D. (2011). Helminths in human carcinogenesis. Cancer Lett. 305, 239-249. doi: 10.1016/j.canlet.2010.07.008

Garrigues, H. J., Demaster, L. K., Rubinchikova, Y. E., and Rose, T. M. (2014). KSHV attachment and entry are dependent on $\alpha \mathrm{V} \beta 3$ integrin localized to specific cell surface microdomains and do not correlate with the presence of heparan sulfate. Virology 464-465, 118-133. doi: 10.1016/j.virol.2014.06.035

Gout, P. W., Buckley, A. R., Simms, C. R., and Bruchovsky, N. (2001). Sulfasalazine, a potent suppressor of lymphoma growth by inhibition of the $\mathrm{x}_{\mathrm{c}}{ }^{-}$cystine transporter: a new action for an old drug. Leukemia 15, 1633-1640. doi: 10.1038/sj.leu.2402238

Greene, W., and Gao, S. J. (2009). Actin dynamics regulate multiple endosomal steps during Kaposi's sarcoma-associated herpesvirus entry and trafficking in endothelial cells. PLoS Pathog. 5:e1000512. doi: 10.1371/journal.ppat. 1000512

Guo, W., Zhao, Y., Zhang, Z., Tan, N., Zhao, F., Ge, C., et al. (2011). Disruption of xCT inhibits cell growth via the ROS/autophagy pathway in hepatocellular carcinoma. Cancer Lett. 312, 55-61. doi: 10.1016/j.canlet.2011.07.024

Gupta, S., Knight, A. G., Gupta, S., Knapp, P. E., Hauser, K. F., Keller, J. N., et al. (2010). HIV-Tat elicits microglial glutamate release: role of NAPDH oxidase and the cystine-glutamate antiporter. Neurosci. Lett. 485, 233-236. doi: 10.1016/j.neulet.2010.09.019

Hahn, A., Birkmann, A., Wies, E., Dorer, D., Mahr, K., Sturzl, M., et al. (2009). Kaposi's sarcoma-associated herpesvirus gH/gL: glycoprotein export and interaction with cellular receptors. J. Virol. 83, 396-407. doi: 10.1128/JVI.01170-08

Haimeur, A., Deeley, R. G., and Cole, S. P. (2002). Charged amino acids in the sixth transmembrane helix of multidrug resistance protein 1 (MRP1/ABCC1) are critical determinants of transport activity. J. Biol. Chem. 277, 41326-41333. doi: 10.1074/jbc.M206228200

Hassman, L. M., Ellison, T. J., and Kedes, D. H. (2011). KSHV infects a subset of human tonsillar B cells, driving proliferation and plasmablast differentiation. J. Clin. Invest. 121, 752-768. doi: 10.1172/JCI44185

Huang, Y., Dai, Z., Barbacioru, C., and Sadee, W. (2005). Cystine-glutamate transporter SLC7A11 in cancer chemosensitivity and chemoresistance. Cancer Res. 65, 7446-7454. doi: 10.1158/0008-5472.CAN-04-4267

Kaleeba, J. A., and Berger, E. A. (2006a). Broad target cell selectivity of Kaposi's sarcoma-associated herpesvirus glycoprotein-mediated cell fusion and virion entry. Virology 354, 7-14. doi: 10.1016/j.virol.2006.06.009

Kaleeba, J. A., and Berger, E. A. (2006b). Kaposi's sarcoma-associated herpesvirus fusion-entry receptor: cystine transporter xCT. Science 311, 1921-1924. doi: $10.1126 /$ science. 1120878

Lo, M., Wang, Y. Z., and Gout, P. W. (2008). The x(c)- cystine/glutamate antiporter: a potential target for therapy of cancer and other diseases. J. Cell. Physiol. 215, 593-602. doi: 10.1002/jcp. 21366

Longnecker, R., and Neipel, F. (2007). "Introduction to the human gammaherpesviruses," in Human Herpesviruses: Biology, Therapy, and Immunoprophylaxis, eds A. Arvin, G. Campadelli-Fiume, E. Mocarski, P. S. Moore, B. Roizman, R. Whitley, and K. Yamanishi (Cambridge: Cambridge University Press).

Ma, Q., Cavallin, L. E., Leung, H. J., Chiozzini, C., Goldschmidt-Clermont, P. J., and Mesri, E. A. (2013). A role for virally induced reactive oxygen species in Kaposi's sarcoma herpesvirus tumorigenesis. Antioxid. Redox. Signal. 18, 80-90. doi: 10.1089/ars.2012.4584

Ma, Q., Cavallin, L. E., Yan, B., Zhu, S., Duran, E. M., Wang, H., et al. (2009). Antitumorigenesis of antioxidants in a transgenic Racl model of Kaposi's sarcoma. Proc. Natl. Acad. Sci. U.S.A. 106, 8683-8688. doi: 10.1073/pnas.0812688106 
Mallery, S. R., Pei, P., Landwehr, D. J., Clark, C. M., Bradburn, J. E., Ness, G. M., et al. (2004). Implications for oxidative and nitrative stress in the pathogenesis of AIDS-related Kaposi's sarcoma. Carcinogenesis 25, 597-603. doi: 10.1093/carcin/bgh042

Manciu, L., Chang, X. B., Buyse, F., Hou, Y. X., Gustot, A., Riordan, J. R., et al. (2003). Intermediate structural states involved in MRP1-mediated drug transport. Role of glutathione. J. Biol. Chem. 278, 3347-3356. doi: 10.1074/jbc.M207963200

Mesri, E. A., Feitelson, M. A., and Munger, K. (2014). Human viral oncogenesis: a cancer hallmarks analysis. Cell Host Microbe 15, 266-282. doi: 10.1016/j.chom.2014.02.011

Myoung, J., and Ganem, D. (2011). Infection of primary human tonsillar lymphoid cells by KSHV reveals frequent but abortive infection of T cells. Virology 413, 1-11. doi: 10.1016/j.virol.2010.12.036

Nabeyama, A., Kurita, A., Asano, K., Miyake, Y., Yasuda, T., Miura, I., et al. (2010). xCT deficiency accelerates chemically induced tumorigenesis. Proc. Natl. Acad. Sci. U.S.A. 107, 6436-6441. doi: 10.1073/pnas.0912827107

Nagasawa, K., Ito, S., Kakuda, T., Nagai, K., Tamai, I., Tsuji, A., et al. (2005). Transport mechanism for aluminum citrate at the blood-brain barrier: kinetic evidence implies involvement of system $\mathrm{X}_{\mathrm{c}}{ }^{-}$in immortalized rat brain endothelial cells. Toxicol. Lett. 155, 289-296. doi: 10.1016/j.toxlet.2004.10.004

Narang, V. S., Pauletti, G. M., Gout, P. W., Buckley, D. J., and Buckley, A. R. (2003). Suppression of cystine uptake by sulfasalazine inhibits proliferation of human mammary carcinoma cells. Anticancer. Res. 23, 4571-4579.

Okuno, S., Sato, H., Kuriyama-Matsumura, K., Tamba, M., Wang, H., Sohda, S., et al. (2003). Role of cystine transport in intracellular glutathione level and cisplatin resistance in human ovarian cancer cell lines. Br. J. Cancer 88, 951-956. doi: $10.1038 /$ sj.bjc. 6600786

Pallis, A. G., and Syrigos, K. N. (2013). Lung cancer in never smokers: disease characteristics and risk factors. Crit. Rev. Oncol. Hematol. 88, 494-503. doi: 10.1016/j.critrevonc.2013.06.011

Pang, X., Panee, J., Liu, X., Berry, M. J., Chang, S. L., and Chang, L. (2013). Regional variations of antioxidant capacity and oxidative stress responses in HIV-1 transgenic rats with and without methamphetamine administration. J. Neuroimmune Pharmacol. 8, 691-704. doi: 10.1007/s11481-013-9454-8

Park, J. Y., Von Karsa, L., and Herrero, R. (2014). Prevention strategies for gastric cancer: a global perspective. Clin. Endosc. 47, 478-489. doi: 10.5946/ce.2014.47.6.478

Patel, S. A., Warren, B. A., Rhoderick, J. F., and Bridges, R. J. (2004). Differentiation of substrate and non-substrate inhibitors of transport system $\mathrm{x}_{\mathrm{C}}{ }^{-}$: an obligate exchanger of L-glutamate and L-cystine. Neuropharmacology 46, 273-284. doi: 10.1016/j.neuropharm.2003.08.006

Pertel, P. E. (2002). Human herpesvirus 8 glycoprotein B (gB), gH, and gL can mediate cell fusion. J. Virol. 76, 4390-4400. doi: 10.1128/JVI.76.9.43904400.2002

Qin, S., Colin, C., Hinners, I., Gervais, A., Cheret, C., and Mallat, M. (2006). System $\mathrm{X}_{\mathrm{C}}{ }^{-}$and apolipoprotein $\mathrm{E}$ expressed by microglia have opposite effects on the neurotoxicity of amyloid- $\beta$ peptide $1-40$. J. Neurosci. 26, 3345-3356. doi: 10.1523/JNEUROSCI.5186-05.2006

Qin, Z., Dai, L., Bratoeva, M., Slomiany, M. G., Toole, B. P., and Parsons, C. (2011). Cooperative roles for emmprin and LYVE-1 in the regulation of chemoresistance for primary effusion lymphoma. Leukemia 25, 1598-1609. doi: 10.1038/leu.2011.144

Qin, Z., Dai, L., Defee, M., Findlay, V. J., Watson, D. K., Toole, B. P., et al. (2013). Kaposi's sarcoma-associated herpesvirus suppression of DUSP1 facilitates cellular pathogenesis following de novo infection. J. Virol. 87, 621-635. doi: 10.1128/JVI.01441-12

Qin, Z., Dai, L., Slomiany, M. G., Toole, B. P., and Parsons, C. (2010a). Direct activation of emmprin and associated pathogenesis by an oncogenic herpesvirus. Cancer Res. 70, 3884-3889. doi: 10.1158/0008-5472.CAN-09-4663

Qin, Z., Freitas, E., Sullivan, R., Mohan, S., Bacelieri, R., Branch, D., et al. (2010b). Upregulation of $\mathrm{xCT}$ by KSHV-encoded microRNAs facilitates KSHV dissemination and persistence in an environment of oxidative stress. PLoS Pathog. 6:e1000742. doi: 10.1371/journal.ppat.1000742

Rappocciolo, G., Hensler, H. R., Jais, M., Reinhart, T. A., Pegu, A., Jenkins, F. J., et al. (2008). Human herpesvirus 8 infects and replicates in primary cultures of activated B lymphocytes through DC-SIGN. J. Virol. 82, 4793-4806. doi: 10.1128/JVI.01587-07
Robe, P. A., Martin, D., Albert, A., Deprez, M., Chariot, A., and Bours, V. (2006). A phase 1-2, prospective, double blind, randomized study of the safety and efficacy of Sulfasalazine for the treatment of progressing malignant gliomas: study protocol of [ISRCTN45828668]. BMC Cancer 6:29. doi: 10.1186/14712407-6-29

Sasaki, H., Sato, H., Kuriyama-Matsumura, K., Sato, K., Maebara, K., Wang, H., et al. (2002). Electrophile response element-mediated induction of the cystine/glutamate exchange transporter gene expression. J. Biol. Chem. 277, 44765-44771. doi: 10.1074/jbc.M208704200

Sato, H., Fujiwara, K., Sagara, J., and Bannai, S. (1995). Induction of cystine transport activity in mouse peritoneal macrophages by bacterial lipopolysaccharide. Biochem. J. 310, 547-551.

Sato, H., Kuriyama-Matsumura, K., Hashimoto, T., Sasaki, H., Wang, H., Ishii, T., et al. (2001). Effect of oxygen on induction of the cystine transporter by bacterial lipopolysaccharide in mouse peritoneal macrophages. J. Biol. Chem. 276, 1040710412. doi: 10.1074/jbc.M007216200

Sontheimer, H., and Bridges, R. J. (2012). Sulfasalazine for brain cancer fits. Expert. Opin. Investig. Drugs 21, 575-578. doi: 10.1517/13543784.2012.670634

Soulier, J., Grollet, L., Oksenhendler, E., Cacoub, P., Cazals-Hatem, D., Babinet, P., et al. (1995). Kaposi's sarcoma-associated herpesvirus-like DNA sequences in multicentric Castleman's disease. Blood 86, 1276-1280.

Taguchi, K., Tamba, M., Bannai, S., and Sato, H. (2007). Induction of cystine/glutamate transporter in bacterial lipopolysaccharide induced endotoxemia in mice. J. Inflamm. (Lond) 4, 20. doi: 10.1186/1476-9255-4-20

Veettil, M. V., Bandyopadhyay, C., Dutta, D., and Chandran, B. (2014). Interaction of KSHV with host cell surface receptors and cell entry. Viruses 6, 4024-4046. doi: 10.3390/v6104024

Veettil, M. V., Sadagopan, S., Sharma-Walia, N., Wang, F. Z., Raghu, H., Varga, L., et al. (2008). Kaposi's sarcoma-associated herpesvirus forms a multimolecular complex of integrins $(\alpha \mathrm{V} \beta 5, \alpha \mathrm{V} \beta 3$, and $\alpha 3 \beta 1)$ and CD98-xCT during infection of human dermal microvascular endothelial cells, and CD98-xCT is essential for the postentry stage of infection. J. Virol. 82, 12126-12144. doi: 10.1128/JVI.01146-08

Xu, D., and Hemler, M. E. (2005). Metabolic activation-related CD147-CD98 complex. Mol. Cell. Proteomics 4, 1061-1071. doi: 10.1074/mcp.M400207-MCP200

Yang, H., Zou, W., Li, Y., Chen, B., and Xin, X. (2007). Bridge linkage role played by CD98hc of anti-tumor drug resistance and cancer metastasis on cisplatin-resistant ovarian cancer cells. Cancer Biol. Ther. 6, 942-947. doi: 10.4161/cbt.6.6.4190

Yang, P., Ebbert, J. O., Sun, Z., and Weinshilboum, R. M. (2006). Role of the glutathione metabolic pathway in lung cancer treatment and prognosis: a review. J. Clin. Oncol. 24, 1761-1769. doi: 10.1200/JCO.2005.02.7110

Zeng, Y., Li, Y., Chen, R. S., He, X., Yang, L., and Li, W. (2010). Overexpression of xCT induces up-regulation of 14-3-3 $\beta$ in Kaposi's sarcoma. Biosci. Rep. 30, 277-283. doi: 10.1042/BSR20090163

Zou, W., Yang, H., Hou, X., Zhang, W., Chen, B., and Xin, X. (2007). Inhibition of CD147 gene expression via RNA interference reduces tumor cell invasion, tumorigenicity and increases chemosensitivity to paclitaxel in HO-8910pm cells. Cancer Lett. 248, 211-218. doi: 10.1016/j.canlet.2006.07.005

Conflict of Interest Statement: The authors declare that the research was conducted in the absence of any commercial or financial relationships that could be construed as a potential conflict of interest.

Received: 26 November 2014; accepted: 30 January 2015; published online: 19 February 2015.

Citation: Dai L, Noverr MC, Parsons C, Kaleeba JAR and Qin Z (2015) xCT, not just an amino-acid transporter: a multi-functional regulator of microbial infection and associated diseases. Front. Microbiol. 6:120. doi: 10.3389/fmicb.2015.00120

This article was submitted to Infectious Diseases, a section of the journal Frontiers in Microbiology.

Copyright (C) 2015 Dai, Noverr, Parsons, Kaleeba and Qin. This is an open-access article distributed under the terms of the Creative Commons Attribution License (CC BY). The use, distribution or reproduction in other forums is permitted, provided the original author(s) or licensor are credited and that the original publication in this journal is cited, in accordance with accepted academic practice. No use, distribution or reproduction is permitted which does not comply with these terms. 